\title{
Tuberculous Pericarditis presenting as Pericardial Tamponade
}

\author{
Maj D G Bowley \\ FRCS, RAMC \\ Specialist Registrar in Surgery \\ Royal Hospital Haslar, Gosport, Hants PO12 2AA
}

Lt Col J Lloyd

MB, ChB, FRCP, RAMC

Consultant Physician

BMH Hong Kong, BFPO I

\section{JRP Gibbons}

MBE, TD, FRCS, FICS, FCCP

Honorary Consulting Surgeon to the Army in Northern Ireland

Royal Victoria Hospital, Belfast

SUMMARY: We present one of the last cases from the British Military Hospital in Hong Kong. A 30 year old woman with pericardial tuberculosis and tamponade is described.

\section{Introduction}

Over the last few years there has been a world wide resurgence in tuberculosis. Tuberculous pericarditis is a rare manifestation of extrapulmonary tuberculosis and tamponade an unusual feature of this disease process.

This case is especially noteworthy as tamponade developed during pregnancy, possibly due to the changing cardiovascular demands of pregnancy.

\section{Case history}

A thirty year-old woman from West Nepal, four months pregnant with her second child, was admitted to hospital with a short history of fatigue and breathlessness associated with fever and central chest pain, worse on inspiration. She was noted to have a markedly raised jugular venous pressure with a low blood pressure and rapid pulse. Chest $\mathrm{X}$-ray revealed a very large heart shadow with clear lungs. A clinical diagnosis of cardiac tamponade was made and pericardial aspiration was attempted. This produced only $50 \mathrm{mls}$ of bloodstained serous fluid.

Because of her deteriorating state a small antero-lateral left thoracotomy was performed, revealing a tense pericardium. Two pericardial windows were made and the removed tissue sent for culture and sensitvity. Granulomata seen on microscopy confirmed the suspicion - of tuberculosis and she was placed on triple therapy. The diagnosis was finally proven by positive culture after six weeks.

She responded well and successfully delivered a normal baby. Her husband is still serving in the Royal Gurkha Rifles.

\section{Discussion}

The World Health Organisation (WHO) estimates that
1722 million persons are infected with tuberculosis. $I_{0} \rightarrow$ most individuals infection is latent or inactive (1). Around 7 8-10 million new cases of tuberculosis occur each yeafD with about 3 million deaths (2).

There has been a world wide resurgence of tuberculosis $\sigma$ mostly in the developing world but also in industrialised countries. Between 1987 and 1993 tuberculosis rates increased by $35.5 \%$ in London compared to $15 \%$ its England and Wales as a whole (3).

Tuberculous pericarditis occurs in $1-4 \%$ of non respiratory tuberculosis $(4,5)$. Infection may reach the pericardium by lymphatic extension from mediastinal lymph nodes, by direct extension from caseous lung tissue or from haematogenous (miliary) spread. Lymphatic extension from mediastinal nodes is considered to be the commonest mode of infection (6).

The pericardial effusion is thought to be due to a hypersensitivity reaction to tuberculoprotein (5). Untreated; fibrocaseous material accumulates within the pericardial space which interferes with ventricular filling. Still later, calcification and true constrictive pericarditis may occur (7).

Clinical manifestations of pericardial effusions are diverse ranging from the asymptomatic with merely an enlarged heart shadow on X-ray to cardiac tamponade (8). Up to $90 \%$ of cases may have features of tamponade, but associated shock is rare (9).

It is known that blood volume in pregnancy is $30 \%$ greater than normal by term and that cardiac output is increased by $30-40 \%$ by 27 weeks (10). We speculate that the increased cardiovascular demands of pregnancy may have provoked pericardial tamponade in this case.

Diagnosis of tuberculous pericarditis may be difficult. Skin testing may be unreliable in the presence of immunosuppression or anergy (5). Strang et al, however, 
found positive Mantoux tests in 239 of 240 patients with tuberculous pericarditis (11). If treatment is delayed marked thickening of the pericardium may occur and is more likely to result in constrictive pericarditis (6). As in a tuberculous pleural effusion only scanty if any mycobacteria may be detected on $\mathrm{ZN}$ staining of pericardial fluid. Confirmation of tuberculous infection is by culture of pericardial aspirate and by culture and histology of pericardium.

Detection of tuberculosis elsewhere in the thorax supports the diagnosis. However active pulmonary tuberculosis may be demonstrated in only $1 / 3$ of cases (11). Indirect methods may be used including detection of adenosine deaminase from pericardial fluid, DNA amplification via the polymerase chain reaction and identification of interferon gamma (6).

Defective immunity may permit recrudescence of previously dormant tuberculosis, while in the past this may have been due to age or alcoholism today the commonest cause is Human Immunodeficiency Virus (HIV) infection (1).

HIV infection is well known to be associated with pericardial disease. AIDS has been identified as the leading diagnosis associated with pericardial effusion in one series in an urban population in the United States (8).

Potential causes of pericardial effusion in AIDS include infection with mycobacteria or cytomegalovirus, or neoplastic processes such as lymphoma, carcinoma and Kaposi's sarcoma (8).

The WHO estimates just over 20 million people are currently infected with HIV and of these 6 million are coinfected with Mycobacterium tuberculosis. People infected with HIV are at increased risk of contracting tuberculosis (3).

Tuberculosis is more common in HIV infection but it is more frequent in its extrapulmonary form (12). Atypical mycobacterial infection is also more common in HIV infection.

Extrapulmonary tuberculosis may occur in greater than $70 \%$ of patients with tuberculosis and pre-existing AIDS or AIDS diagnosed soon after the diagnosis of tuberculosis (12), indeed extrapulmonary tuberculosis has been an AIDS defining illness since 1987.

Primary pericardial effusion in the absence of HIV infection may also be idiopathic, or associated with toxoplasma, bacterial pneumonia, systemic lupus erythematosis, rheumatoid arthritis, thyroid disorders or dissecting aortic aneurysm (13).

Treatment of pericardial tuberculosis should be with 6-9 months of standard chemotherapy $(5,6)$. The place of steroids in the treatment of pericardial tuberculosis has become clearer since the publication of large randomised series from South Africa (11,7). Corticosteroids increase the rate of clinical improvement during antituberculosis chemotherapy and probably reduce the risk of death and the need for pericardiectomy.

Multidrug resistant tuberculosis is well recognised, however between 1982 and 1991 only $0.6 \%$ of primary isolates were resistant to isoniazid and rifampicinz Provisional figures for 1994 show $2.8 \%$ of isolates from the North Thames region and $1.4 \%$ of isolates from the Southo Thames region are multidrug resistant (3).

Pericardiocentesis should be performed for diagnostice? purposes and for symptomatic effusions. This can be repeated but for rapidly recurring effusions surgery is indicated. This may be undertaken via a subxiphoig approach, via an antero-lateral thoracotomy of thoracoscopically (14).

Prior to the introduction of antibiotics, tuberculou市 pericarditis had a mortality rate of $80-90 \%(4,5)$. In the modern setting Strang et al reported mortality of 3\% in patients treated with steriods and anti-tuberculous drugs and a mortality of $14 \%$ in those given anti-tuberculous chemotherapy only (11). Hugo-Hamman and colleagues? recently reported a series of 44 cases of tuberculous pericarditis in children from Cape Town, recovery was complete in 43 cases with 1 unrelated death. Only 5 of the children required pericardiectomy (9).

\section{Conclusion}

All clinicians need to be aware of tuberculosis and howb it may present at various sites. Population movement and the rise in HIV infection have led to an increased incidege of tuberculosis. Pericardial tuberculosis is rare but shoull $\mathbb{E}$ be considered in the differential diagnosis of all cases pericarditis.

We believe that in this case the extra demands placedon the cardiovascular system during pregnancy precipita pericardial tamponade.

Medical therapy for tuberculous pericarditis is usu successful but surgical approaches allow drainage of flutrd histological diagnosis and obviate the need for repeate pericardiocentesis.

\section{REFERENCES}

1. WATTERS DAK. Surgery for tuberculosis before an $\$$ after human immunodeficiency virus infection: tropical perspective. BrJ Surg 1997; 84: 8-14.

2. The global challenge of tuberculosis, Editorial. Lance 1994; 344: 277-278.

3. COKER R, Miller R. HIV associated tuberculosis. $B$ Med J 1997; 314: 1847.

4. Quale JM, Lipschik GY, Heurich AE. Managemen? of tuberculous pericarditis. Ann Thorac Surg 1987; 43? 653-655.

5. FOWLER NO. Tuberculous pericarditis. Jama 1991 266: 99-103.

6. Coulter JBS, Walsh K, King SJ, Shears Po Tuberculous pericarditis in a child. I Infect 1996; 32? 157-160.

7. Strang JiG, Kakaza HHS, Gibson DG, Girling DJ NunN AJ, Fox W. Controlled trial of prednisolone as adjuvant in treatment of tuberculous constrictiva pericarditis in Transkei. Lancet 1987;Dec 19: 1418^u 1422.

8. Flum DR, McGinn JT, Tyras DH. The role of the

\section{.}


Pericardial window in AIDS. Chest 1995; 107: $1522-$ 25.

9. Hugo-hamman CT, Scher H, De Moor MMA. Tuberculous Pericarditis in children: a review of 44 cases. Paediatr Infect Dis J 1994; 13: 13-18.

10. GuYTon AC, Hall JE. Textbook of Medical Physiology. 9th ed. London: WB Saunders \& Co., 1996: 1044.

11. Strang JIG, Kakaza HHS, Gibson DG, et al. Controlled clinical trial of complete open surgical drainage and of prednisolone in treatment of tuberculous pericardial effusion in Transkei. Lancet 1988; Oct 1: 759-764.
12. Serrano-Heranz, Camino A, Vilacosta I, LopezCastellanos A, Roca V. Tuberculous cardiac tamponade and AIDS. Euro Heart $J$ 1995; 16: 430432.

13. Zayas R, ANGUita M, TORRES F, et al. Incidence of specific etiology and role of methods for specific etiologic diagnosis of primary pericarditis. Am $J$ Cardiol 1995; 75: 378-382.

14. Mack MJ, Aronoff RJ, AcufF TE, Douthit MB, BOWMAN RT, RYAN WH. Present role of thoracoscopy in the diagnosis and treatment of diseases of the chest. Ann Thorac Surg 1992; 54: 403-409. 